\title{
Towards an Ontology-Based Decision Support System for Syndrome-Differentiation and Treatment of Psoriasis Vulgaris in Traditional Chinese Medicine
}

\author{
Hai Long \\ Universitat Leipzig \\ Zhe Wang \\ Universitat Leipzig \\ Yidi Cui
}

China Academy of Chinese Medical Sciences

Junhui Wang

China Academy of Chinese Medical Sciences Guanganmen Hospital

\section{Bo Gao}

China Academy of Chinese Medical Sciences

Chao Chen

China Academy of Chinese Medical Sciences

Heinrich Herre

Universitat Leipzig

Yan Zhu ( $\nabla$ zhuyan166@126.com )

China Academy of Chinese Medical Sciences https://orcid.org/0000-0002-5592-8258

Technical advance

Keywords: clinical guideline, knowledge base, GFO, ontology, TCM, CBR, fuzzy logic, psoriasis vulgaris.

Posted Date: July 6th, 2020

DOI: https://doi.org/10.21203/rs.3.rs-38383/v1

License: (c) (i) This work is licensed under a Creative Commons Attribution 4.0 International License. Read Full License 


\section{Abstract}

Background: Psoriasis is a chronic, non-communicable, painful, disfiguring and disabling disease, which is not curable and strongly declines the patients' quality of life (QoL). However, diagnose and treatment in traditional Chinese medicine (TCM) based on syndrome differentiation has been used in practice for a long time and has also achieved some effect. Though, up to now, only few studies are available reporting on the use of semantic technologies and pertain to knowledge systems that use TCM-syndrome differentiation for information retrieval and automated reasoning. Nowadays, the diagnosis in TCM relies mainly on the personal expertise and clinical experience of the doctors. For various reasons, misdiagnoses or missed diagnoses cannot be completely excluded, leading to unexpected results.

Methods: Firstly, we developed a domain ontology for syndrome differentiation of psoriasis vulgaris. For this purpose, we used the ontology editor Protégé and applied a top-down approach which adopts the framework of general formal ontology (GFO) and its middle-level core ontology GFO-TCM. Furthermore, we implement a prototype which is based on this ontology. Additionally, we also used a case-database for CBR (Case Based Reasoning) combined with fuzzy pattern recognition.

Results: A prototype for diagnosis and treatment of psoriasis vulgaris, named ONTOPV-system, is proposed which is based on the principle of syndrome differentiation and treatment in TCM; this system realizes an expert-assisted decision support method which relies on a domain ontology, uses fuzzy logic reasoning, and case retrieval and is intended to support clinical diagnostic decisions for TCM practitioners.

Conclusions: We designed and implemented a prototype for psoriasis diagnosis in terms of syndrome differentiation. The system can not only realize the basic functionalities of data collection, querying, browsing and navigation, but also support rule-based knowledge reasoning and customize approximate reasoning based on CBR through fuzzy logic, which can provide users with clinical decision support for TCM syndrome differentiation in diagnosis of psoriasis. In addition, it comprises a domain knowledge base of psoriasis, which is developed based on the GFO framework with good extensibility.

\section{Background}

Ontologies play an increasing role in the field of knowledge representation; actually, they present the most advanced techniques to analyze, conceptualize and axiomatize the knowledge of a domain of interest. Knowledge exhibits various levels of abstraction; we may distinguish general knowledge, presented often by rules and formal axioms, from knowledge about individual cases. Furthermore, ontologies are more and more combined with other methods of artificial intelligence, notably with techniques of machine learning. A particular interesting method is case-based reasoning (CBR) which originates from cognitive psychology and is today a hot subtopic of artificial intelligence. Case based reasoning is a form of analogical reasoning: when encountering a novel problem, one searches for an already solved case which is closest to the problem. Then, the solution of the known case is adapted to a solution of the new 
problem. After the new problem is solved, the revised case will be recorded and added as an experience [1]. Compared with western medicine, the diagnosis and treatment in traditional Chinese medicine (TCM) pursues a personalized approach. According to the patient's behavior of yin and yang, of cold and heat, as well as of the course of the disease, a corresponding interventional plan will be proposed, using a large number of typical and different cases. The CBR's process is similar to that of TCM's diagnosis process which uses previous experience ${ }^{[2]}$ and relies on the thinking way in terms of imagery and gets help from analogical reasoning ${ }^{[3-4]}$. Therefore, the unique characteristics of TCM about personalized diagnosis and treatment makes itself more suitable for application of CBR. Until now, CBR has been gradually applied into the field of TCM, particularly focusing on the design of expert systems and clinical decision support systems (CDSS). For example, Yang Li et al. established a TCM expert system based on CBR ${ }^{[5]}$; Peng et al. developed a syndrome differentiation and treatment system in TCM ${ }^{[6]}$. Wang et al. proposed a CBRbased TCM expert system combined with fuzzy rule-based reasoning ${ }^{[7]}$. These works show that the reasoning mechanisms for CDSS of TCM, based on CBR, are becoming more and more mature. However, due to the insufficient amount of cases, poor data quality, and limited clinical applications, most of these systems are not capable to provide sufficient support for an accurate clinical decision-making in TCM. And the lack of consideration of functionality and expansibility of knowledge base has also weakened the accuracy and reliability of the embedded information needed for clinical decision support.

Psoriasis, classified by the WHO as a severe disease ${ }^{[8]}$, is a chronic, non-communicable, painful, disfiguring, non-curable and disabling disease, which heavily affects the patients' quality of life (QoL). However, diagnosis and treatment with TCM based on syndrome differentiation has a long practical history and achieved some effect. ${ }^{[9-11]}$. According to TCM theory, Psoriasis is caused by many factors, such as blood deficiency, blood heat, dryness and wind, as well as lack of nutrition. It can be divided into different syndrome types based on characteristics of clinical symptoms. Accordingly, different treatment principles and methods shall be applied. The diagnosis in TCM is based on the comprehension of manifestation of pulse, tongue coating, complexion, physical constitution and other signs and symptoms, some of which are certain, while many of them are fuzzy, ambiguous, nonlinear and uncertain ${ }^{[12]}$. In the process of diagnosis, TCM doctors often rely more on their own knowledge and experience to find solutions, rather than using logical reasoning. Therefore, it is difficult to establishing a TCM expert system, due to uncertainty of knowledge representation, data acquisition and the lack of logic reasoning. The complexity and fuzziness of TCM-knowledge leads to new challenges because there is the need to develop new forms of on knowledge representation and reasoning mechanism.

In clinical practice, TCM doctors make the diagnosis of psoriasis mainly on their professional knowledge and clinical experience. Young and inexperienced doctors are prone to make misdiagnosis or to overlook diagnoses, resulting in the lower-than-expected curative effect. In this paper we propose a prototypical TCM-decision support system for diagnosing and treating of psoriasis, called ONTOPV, which is based on a domain ontology and which uses CBR (Case-Based Reasoning) combined with fuzzy pattern recognition. This system exploits the valuable and extensive heritage of TCM knowledge. 


\section{Methods}

\section{Architecture of the ONTOPV system}

Up to now, many ontology-based clinical decision support systems for different types of diseases have been developed and achieved convincing results in the clinical practice ${ }^{[13-16]}$. This valuable work provides us with many inspirations. In this paper we present a prototype towards an expert-system, named ONTOPV, which is based on an ontology of TCM-diagnostic knowledge of psoriasis vulgaris, and which uses a case-database for CBR (Case Based Reasoning). This system is intended to help doctors or other users to diagnose psoriasis related diseases, and to provide clinical decision support if necessary. The prototype of the ONTOPV-system is elaborated to realize at least the following three procedures:

1. Diagnosis of $P V$ : The doctor gathers the required information by querying and observing the patient, including age, gender, lifestyle, family history, signs and symptoms. Then, the patient's main complaint and the acquired information is put into a template, the content of which is transformed as input to the rule basis and diagnosis ontology. The inference engine derives possible diagnoses which is provided to the doctor who receives advice for diagnosis and treatment.

2. Development ofintervention plans: according to the recommended diagnostic results, an intervention plan is (semi-) automatically generated for the patient diagnosed. This intervention plan includes some TCM treatments and/or common therapies of Western medicine, like topic- and photo therapy, etc. Further, a personalized care plan on demand will be developed and a solution is recommended for the patients.

3. Information Retrieval: with a semantic querying mechanism, users may access the system to ask questions about the Information or required Data that are stored in the Ontology and in the CBR based KB of PV. Then, the answers together with relevant graphic displays will be returned to the user.

The following figure gives an overview of the system. It shows that the diagnostic information collected by the doctor from the patient, such as symptoms, signs, skin lesion area, and other diagnostic information, is input into the knowledge based system as input parameters, compared with the syndrome type, stored in the ontology, and matched against the customized rules in the SWRL rule base. If the given prerequisites are met, one or more inference rules are triggered to obtain the corresponding diagnosis result and the related treatment plan. The input is also compared with the past cases in the case database. If there are matching case records, the cases with higher similarity are extracted as reference case and provided to the user for comparative analysis.

\section{Construction of domain ontology for syndrome differentiation of psoriasis vulgaris}

In our work we developed a domain ontology for syndrome differentiation of psoriasis vulgaris. For this purpose, we used the ontology editor Protégé and applied a top-down approach which adopts the 
framework of general formal ontology (GFO) and its middle-level core ontology GFO-TCM described in ${ }^{\text {[17- }}$ 20].

\section{Basic conceptual hierarchy}

For the construction of the basic conceptual hierarchy we used various documents, such as EvidenceBased Clinical Practice Guideline of TCM for psoriasis vulgaris (2013) ${ }^{[21]}$ (hereafter referred to Guideline 2013), the WHO Global Report on Psoriasis $(2016)^{[8]}$, the Guideline for the diagnosis and treatment of psoriasis in China (2018 simplified edition) ${ }^{[22]}$, etc.

Furthermore, we used TCM-related documents about psoriasis vulgaris which provide information about the classification of psoriasis, symptoms (primary symptoms, secondary symptoms and combined symptoms), syndrome types, diagnosis, treatment methods, prescriptions, drug ingredients, drug compatibility, etc. Some basic semantic types have been obtained from the guidelines and other related literature, such as disease name; basic syndrome types (ㅁ): blood heat syndrome, blood dryness syndrome, blood stasis syndrome; simultaneous syndromes; basic symptoms including main symptoms, minor symptoms; methods of treatment; recommended formulas and drugs; pharmaceutical ingredients

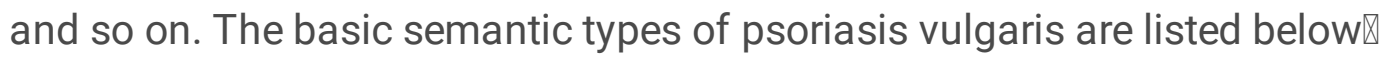

-Health Problems: The psoriasis-related health problems are mainly divided into 3 categories, namely, disease, symptom, (incl. main symptoms, minor symptoms, accompanied symptoms), and syndrome or pattern;

-Formula: A formula or prescription usually contains some medicinal components, used for treating an illness, such as psoriasis vulgaris.

-Drug/Medicinal: Usually referring to those medicinal substances recorded in Chinese materia medica but also to some medicaments of western medicine.

-TCM Diagnose for syndrome differentiation: According to the Guideline (2013), each type of syndrome of psoriasis vulgaris has a set of corresponding symptoms, which can provide a guideline for the diagnosis of TCM syndrome differentiation.

-Method of treatment: The method of treatment is composed of the conventional therapies in TCM and western medicine, such as: TCM prescriptions and drugs, medicated bath, biological therapy, topical therapy, systemic therapy, phototherapy, etc.

-Participant/Person: Such as doctors, patients, etc.

-Compatibility and Caution: Prescribe the medicinal composition and dosage of prescriptions, special instructions and contraindications between certain medicinal or chemical ingredients.

\section{Instantiation}


According to the instructions in the Guideline (2013), the corresponding instantiated entities are established for the basic semantic types from the classification in the ontology, such as instances for major symptoms, minor symptoms, combined symptoms, prescriptions, drugs, pharmaceutical ingredients, treatment methods, etc.

\section{Object- and datatype properties}

Finally, the relationship between different entities in the TCM diagnosis ontology for psoriasis vulgaris needs to be clarified. About 20 common binary object properties that relate individuals to individuals have been specified. These include: annotate (a disease can be annotated by some syndromes): according to the guideline 2013, PV can be annotated by several zheng/syndromes, including some basic zhengs and combined zhengs, due to its different onset process or observed symptoms. Further object properties are: hasComponent (a formula has certain drug components); hasAdverseReaction (some drug components may cause adverse reaction with each other), diagnosed (a patients could be diagnosed with psoriasis vulgaris); hasPattern (for example, the patient's syndrome type is blood-heat type); hasPrescription (some prescriptions can be proposed according to some kind of certain syndrome); recommended (some treatment methods could be recommended for a given diagnosis); hasSymptom (a syndrome type may have some symptoms). Through these built-in object properties, individuals which belong to different concepts in the ontology are correlated to each other, and stored in the OWL ontology in the form of RDF triple as object property assertions. Then, some basic datatype properties that relate individuals to literal data are built as well, such as hasBSA that means Body Surface Area, i.e. the size of body surface occupied by psoriasis; hasTherapyPeriode; hasDosage; etc.

\section{Construction of the rules base}

The rules of diagnosis-based syndrome differentiation and treatment must take into account specific regulations for diagnosis and medication in the evidence-based guidelines of TCM for psoriasis vulgaris $^{[21]}$. For example, a corresponding treatment mechanism is established for the patients' observed symptoms as defined by the guidelines to ensure compliance with the standard operation required by the guidelines. In the ONTOPV related TCM diagnosis ontology, the reasoning rules are constructed for diagnosing certain syndrome types in terms of corresponding sets of symptoms. And there are further rules which are used to check the compatibility of drug components and allows to query whether some given components may cause side effects due to drug interactions. Using the rule base, a reasoning process can be performed the results of which provide diagnoses and /or treatment suggestions according to the patient's symptoms. The followings are examples and explanations of some rules in the SWRL rules case.

\section{Rules for the assessment of PV}

Patient(?a) $\wedge$ diagnosed(?a, psoriasis_vulgaris) $\wedge$ hasBSA(?a, ?c) $\wedge$ swrlb:lessThanOrEqual(?c, 0.03) $->$ hasAssessmentOfSeverity(?a, "mild") 
A patient (a) with PV who has a skin lesions less or equal than $3 \%$ of the Body Surface Area (BSA) should be evaluated as "mild" in terms of degree of severity.

Patient(?a) $\wedge$ diagnosed $(? a, p v) \wedge$ hasBSA(?a, ?c) $\wedge$ swrlb:greaterThan(?c, 0.03) $\wedge$ swrlb:lessThan(?a, $0.1)$-> hasAssessmentOfSeverity(?a, "moderate")

A patient (a) with PV who has a skin lesions greater than $3 \%$ of BSA and less than or equal to $10 \%$ of BSA should be evaluated as "moderate" in terms of degree of severity.

Patient(?a) $\wedge$ diagnosed(?a, pv) $\wedge$ hasBSA(?a, ?c) $\wedge$ swrlb:greaterThanOrEqual(?c, 0.1) $\rightarrow$ hasAssessmentOfSeverity(?a, "severe")

A patient (a) with PV who has a skin lesions greater than $10 \%$ of BSA should be evaluated as "severe" in terms of degree of severity.

\section{Rules for syndrome differentiation of PV}

Patient(?a) $\wedge$ diagnosed(?a, pv) $\wedge$ hasSymptom(?a, bright_red_lesions) $\wedge$ hasSymptom(?a, continusously_increased_rashs) $\wedge$ hasSymptom(?a, yellow_urine) $\rightarrow$ denote(pv, syndrome_of_heat_in_blood)

A patient (a) with psoriasis vulgaris who has main symptoms of red lesions and continuously increased rash and has a minor symptom of yellow urine, the pattern would be assigned to a "syndrome of blood heat" खाया

Patient(?a) $\wedge$ diagnosed(?a, pv) $\wedge$ hasSymptom(?a, pink_lesions) $\wedge$ hasSymptom(?a, dry_scales $) \wedge$ hasSymptom(?a, dry_mouth_and_throat) -> denote(pv, syndrome_of_blood_dryness)

A patient (a) with psoriasis vulgaris who has main symptoms of red lesions and continuously increased rash and has a minor symptom of dry mouth and throat, then the pattern would be assigned to a "syndrome of blood dryness(미)".

\section{Rules for prescription:}

Patient(?a) $\wedge$ diagnosed $(? a, p v) \wedge$ Pattern(?b) $\wedge$ denote(pv,syndrome_of_heat_in_blood)-> hasPrescription(?b, decoction_of_cooling_blood_and_toxin_resolving)

A patient (a) with psoriasis vulgaris who has been ascribed to has a syndrome of heat in blood would be

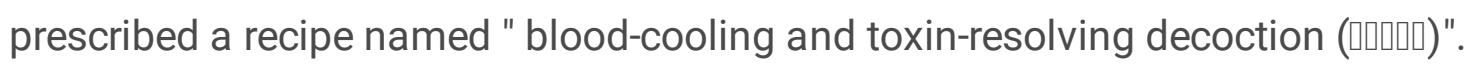

Patient(?a) $\wedge$ diagnosed(?a, pv) $\wedge$ Pattern(?b) $\wedge$ denote(?b,syndrome_of_blood_dryness) $->$ hasPrescription(?b, decoction_of_nourishing_blood_and_toxin_resolving)

A patient (a) with psoriasis vulgaris who has been ascribed to a syndrome of blood stasis would be

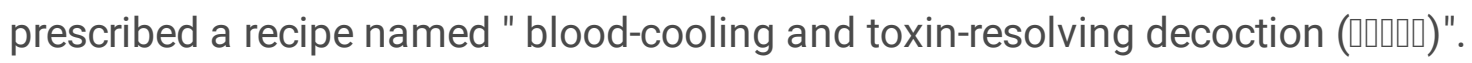

\section{Rules for treatment of psoriasis:}


Patient(?a) $\wedge$ diagnosed(?a, ?b) $\wedge$ Topical_Therapy(?c) $\wedge$ hasAssessmentOfSeverity(?a, "Mild") $->$ recommend(?b, ?c)

A patient with psoriasis who has been evaluated as "Mild" in terms of severity of skin lesions should be recommended to receive some topical therapies.

Patient(?a) $\wedge$ diagnosed(?a, ?b) $\wedge$ Biologics(?c) $\wedge$ hasAssessmentOfSeverity(?a, "Severe") $\wedge$ hasDLQI(?a, ?d) $\wedge$ swrlb:greaterThan(?d, 20) $\rightarrow$ recommend(?b, ?c)

A patient with psoriasis who has been evaluated as "Severe" in terms of the severity of skin lesions, and has a Dermatology Life Quality Index (DLQI), which is greater than 20, should be recommended to receive some biologics.

\section{Rules for drugs adverse reaction}

drugs(?a) $\wedge$ drugs(?b) $\wedge$ hasComponent(?a, danshen_root) $\wedge$ hasComponent(?b,aluminum_hydroxide)-> hasAdverseReaction(?a, ?b)

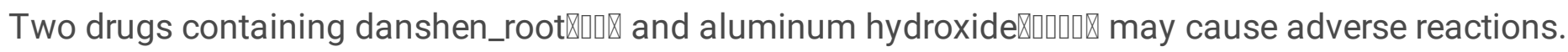

$\operatorname{drugs}(? a) \wedge \operatorname{drugs}(? \mathrm{~b}) \wedge$ hasComponent(?a, Sichuan_fritillary_bulb) $\wedge$ hasComponent(?b, prepared_common_monkshood_branched_root)-> hasAdverseReaction(?a, ?b)

Two drugs containing Sichuan_fritillary_bulb (미) and prepared_common_monkshood_branched_rootखाय may cause adverse reactions.

\section{Case-based reasoning using fuzzy logic}

As mentioned above, a diagnosis of TCM is usually based on the comprehension of manifestation of pulse, tongue coating, complexion, physical constitution and other signs and symptoms, some of which are certain while more of them are fuzzy, ambiguous, nonlinear and uncertain. Hence, in the process of diagnosis, TCM doctors often more rely on their own knowledge and experience rather than logical reasoning to find solutions. Therefore, the difficulty of establishing a TCM expert system lies in the uncertainty of knowledge representation, data acquisition and logic reasoning. The complexity and fuzziness of knowledge faces new challenges and requirements to knowledge representation and reasoning mechanism.

In the case of psoriasis vulgaris, for any possible diagnosis and treatment in view of syndrome differentiation, TCM doctors must firstly identify the patient's symptoms, in order to obtain the corresponding diagnosis results. This could be considered as a kind of pattern recognition. According to M. Friedman and A. Kandel ${ }^{[23]}$, a pattern is an abstract object which is inspected for the recognition process. And we usually regard a pattern as a schematic description of an object which we want to recognize. In most cases, a pattern recognition problem is a problem of discrimination between different populations, namely, a kind of cognitive classification of the object being studied. This classification is performed on the premise of known patterns, that is, the population can be divided into several types, as a set of standard patterns, to identify a specific object to which pattern it belongs, say, which type. 
We took an example from the evidence-based clinical guidelines for psoriasis vulgaris in TCM (2013), according to the diagnostic criterion of blood-heat syndrome, which may have two main symptoms of bright red lesions, continuously increased rashes, and four minor symptoms including irritability and upset, yellow urine, red tongue, rapid pulse. As long as the patient has all major symptoms and at least one minor symptom, the syndrome can be determined. The same principle could be applied to the identification of blood dryness syndrome, except that the latter symptoms can be expressed as major symptoms of pink lesions, dry scales and minor symptoms of dry mouth, pale tongue, thready pulse. If the patient is observed with some symptoms of blood heat syndrome, combination with some symptoms of blood dryness syndrome, the question is how to identify this patient. This case can be observed quite often in practice, because the onset of this disease is a gradual process according to the theory in TCM. In the early stage of this disease, it is mostly manifested as blood heat syndrome, and mostly blood dryness syndrome in the middle stage, but mostly blood stasis syndrome in the late stage. It can also be manifested as other types of syndrome simultaneously. In TCM sometimes decisions cannot be easily made on the basis of two-valued logic. ${ }^{[24]}$ Hence, the problem about pattern recognition in a process of syndrome differentiation can be considered as a more-or-less type rather than yes-or-no type which pertains to the topic of fuzzy pattern recognition.

In this paper, inspired by Zadeh's fuzzy set theory ${ }^{[25-27]}$, a mechanism of information retrieval is proposed, which integrates ontology and CBR using fuzzy pattern recognition This integration provides automatic reasoning and semantic retrieval in line with the norms of TCM evidence based clinical guidance for psoriasis vulgaris, and additionally also simulates the thinking mode of experts based on fuzzy logic. Meanwhile, a solution is carried out to find a similar case from the case base using the proposed method of lattice degree of nearness ${ }^{[28][23]}$. In the ONTOPV prototype, the user can input data, collected from patients as input parameters into the system. For example: main symptoms, minor symptoms, personal information (age, gender, past history, family history, etc.), chief complaint (which means subjective description of the patient), etc.

Then the system processes these data with searching rules defined in the SWRL rules base. If the given preconditions are met, one or more rules are triggered, and subsequently the corresponding standard diagnosis results and treatment scheme will be obtained. At the same time, it can also be compared with the past cases stored in the case database. If there is a matching case record, the case with higher similarity will be extracted as the reference case and provided to the user for comparative analysis. The workflow of case retrieval in ONTOPV is shown in Figure 2.

Firstly, the cases of patients can be considered as a pattern space $A=\left(a_{1}, a_{2} \ldots a_{m}\right)$, where $a_{i}, 1 \leq i \leq m$ are vectors in $\mathrm{Rn}$, i.e. each pattern is characterized by $\mathrm{n}$ features. For example, each case can be characterized by gender, age, patient's chief complaint, symptoms, Western medicine diagnosis, TCM diagnosis, syndrome type, treatment method, recommended medication, and quantitative score of each syndrome type based on the comprehensive score according to the membership function defined. 
Suppose the universe of discourse $U=\{$ several patient cases $\}$, the diagnostic characteristics of the patient-cases include several symptoms, which need to be diagnosed and classified according to the color of the lesion, the nature of the lesion, tongue- and pulse manifestation, urination, complexion, and chief complaint. Thus, we can get the pattern space of syndrome $S=\{$ blood heat syndrome, blood stasis syndrome, blood dryness syndrome, combined syndrome\}, and the combined syndromes can be further divided into wind, dampness, and heat poisoning. Among them, the pattern of blood heat-, blood stasis-, blood dryness-, and combined syndrome can be considered as fuzzy set. To determine which type syndrome the patient presents with, a TCM doctor must firstly determine which features, say symptoms go to this classification. This problem can be measured by using the degree of membership and of similarity between two fuzzy sets $A$ and $B$, which is discussed below.

Using the principle of maximum membership in fuzzy set, a simple membership function can be firstly established to determine which basic syndrome type a patient has. According to the guideline, a basic syndrome type can be defined. If a patient exhibits two major symptoms and at least one minor symptoms, then the diagnosis is true, which means the patient's symptoms have full membership degree with this syndrome type. Accordingly, a simple membership function can be established.

We have assigned a weight value to each symptom, including the main- and the minor symptom, For example, there are altogether 2 main symptoms and 4 minor symptoms in the blood-heat syndrome. Then a weight value of 0.3 is assigned to each main symptom and 0.1 to each minor symptom. Similarly, we assign a weight value to each symptom in every syndrome type, so that we can establish a simple membership function to judge a given case of a patient, membership degree of this patient's syndrome type. Then we only need to add the weights of the patient's symptoms to estimate the degree of membership in the class of a syndrome type he may have.

Suppose we have a fuzzy set of symptoms and syndrome of a patient in the case database $P=\{b l o o d$ fever, bright red lesions, dry scales, slow pulses, pale tongue, yellow urine, heavy head\} corresponding to each basic syndrome of blood heat, blood dryness, blood stasis due to the defined membership function above, a fuzzy vector of the patient $p=(1,0.5,0.3)$ which may be interpreted that the patient's symptom set has respective grade of membership: 1 (true) for blood heat syndrome of and 0.5 (possible) for blood dry syndrome type of and 0.3 (less possible)for combined syndrome wet.

Let us assume there are two reference cases $A$ and $B$ in the case base, and both correspond to the abovementioned three syndrome types in terms of a grade of membership:

Given as fuzzy vector $a=(0.6,0.4,0.2)$, with the symptom set $\{$ Qi deficiency, bright red lesions, upset, red tongue, string pulse, dry scales, dry mouth, dizziness\}

And fuzzy vector $b=(0.7,0.1,0.1)$ with the symptom set $\{$ blood heat accumulation, white scales, red skin lesions, thin and white tongue fur, dry mouth, slippery pulse\} 
Using the method of lattice degree of nearness proposed by P.Z.Wang ${ }^{[25]}$, the similarity between two fuzzy sets can be defined by the following expression:

$$
\sigma_{0}(A, B)=\frac{1}{2}[A \bullet B+(1-A \oplus B)]
$$

Where $A \bullet B$ and $A \oplus B$ are the inner and outer products of $\mathrm{A}$ and $\mathrm{B}$, i.e.

$$
\begin{aligned}
& A \bullet B=\max \left\{\min \left[X_{A}(x), X_{B}(x)\right], x \in X\right\} \\
& A \oplus B=\min \left\{\max \left[X_{A}(x), X_{B}(x)\right], x \in X\right\}
\end{aligned}
$$

Then the inner product and the outer product of $p$ and a are 0.6 and 0.3 respectively; while the inner product and the outer product of $p$ and $b$ are 0.7 and 0.3 respectively. The lattice distance between the two is 0.65 and 0.7 . Obviously, the case of $P$ and $B$ is closer. Therefore, $B$ can be taken as an approximate case.

\section{Results And Discussion}

The ONTOPV prototype can graphically display the knowledge, related to psoriasis vulgaris which includes: knowledge of the disease, syndrome types, related symptoms, diagnosis and treatment methods, prescription, drugs component, which all have been built in the ontology. Then, some practical functions, such as ontology reasoning, case retrieval, semantic query, and medication alert can be implemented.

\section{Ontology reasoning}

According to the patient input information, including main symptoms and minor symptoms, etc., evaluation and analysis can be performed. If the input symptoms can meet the diagnostic criteria of the syndrome type, the system will prompt the user with the corresponding diagnosis suggestion for reference. For example, after imputing the main symptoms to a patient, including bright red lesion, continuously increased rashes, and the minor symptoms, including irritability and upset, yellow urine, the system obtains the inferred results of a blood-heat syndrome (미) through reasoning over ontology, and output the corresponding medication recommendations: blood-cooling and toxin-resolving decoction (पत्य ه).

\section{Case retrieval}

In the case database of the ONTOPV prototype, by using the above-mentioned method of fuzzy pattern recognition, the input symptoms and signs of a patient can also be used to find a similar case from the 
case database. For example, we take the same input symptoms as an example for ontology reasoning, as a consequence, the no. 16 case is matched with maximal similarity and output as query results which is shown in Figure 4.

\section{Semantic query and visualization}

In the ONTOPV prototype we can perform a semantic query over the knowledge base that is built in the ontology.

For example, if we query the prescription of blood-cooling and toxin-resolving decoction in the knowledge base, the system will display its ingredients, dosage and usage as shown blow.

\section{Medication alert}

In the ONTOPV prototype, the user is allowed to input some prescription. If there are two drugs containing some ingredients that may cause adverse reactions, the system will alert the users to the adverse reactions of the drug to the patient. For example, if a doctor prescribes a combination drug product of

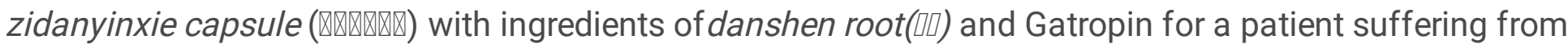
indigestion, the system will prompt that the two drugs due to having ingredient of aluminum hydroxide may bring about adverse reactions and should be used cautiously.

\section{Conclusions And Future Research}

As described in this paper, we designed and implemented a prototype for psoriasis diagnosis based on syndrome differentiation. The system can not only realize the basic functionalities of data collection, querying, browsing and navigation, but also support rule-based knowledge reasoning and customize approximate reasoning based on CBR through fuzzy logic, which can provide users with clinical decision support for TCM syndrome differentiation in diagnosis of psoriasis. In addition, it comprises a domain knowledge base of psoriasis, which is developed based on the GFO framework with good extensibility. Through visualization of the knowledge graph and the query interface, doctors can quickly retrieve the needed information from the knowledge base. Furthermore, the system also provides a module for detection of medication conflicts, based on knowledge-based reasoning, which can assist doctors to ensure drug-use safety and reduce drug-induced risks. The future works will include collecting more electronic medical records and cases, extending query and reasoning for all syndromes of psoriasis, as well as integrating the clinical diagnosis and treatment knowledge of TCM and Western medicine. These methods will be extended to other TCM syndromes, and for this purpose we need an ontology of Zhengtypes and tools to organize and compute syndromes/Zheng and to extract semantic information from EHR (electronic health care records), according to ${ }^{[29]}$.

\section{List Of Abbreviations}


CBR: case-based reasoning; CDSS: clinical decision support systems; GFO: General Formal Ontology;

TCM: Traditional Chinese Medicine;

\section{Declarations}

\section{Ethics approval and consent to participate}

Not applicable.

\section{Consent to publication}

Not applicable.

\section{Availability of data and materials}

The data that support the findings of this study are available from the corresponding author on request.

\section{Competing interests}

The authors declared that they have no competing interests.

\section{Funding}

This work was partially supported by National Natural Science Foundation of China [61701546], National Key R\&D Program of China (2019YFC1710400; 2019YFC1710401) and the Fundamental Research Funds for the Central public welfare research institutes [ZZ13-YQ-126, ZZ13-YQ-021]. The publishing costs for this manuscript were provided by National Natural Science Foundation of China [61701546].

\section{Authors' Contributions}

$\mathrm{HL}$ wrote the main manuscript text. $\mathrm{HL}$ and $\mathrm{YZ}$ conceived the idea and designed the study. ZW contributed to the visualization. YC, BG, JW and CC participated in case gathering and interpretation. $\mathrm{HH}$ guided the study and revised the manuscript. All authors reviewed and approved the manuscript.

\section{Acknowledgements}

We appreciate Mrs. Huang Xiao Fang very much for checking and revising the manuscript.

\section{References}

1. M. Richter (auth.), M.Lenz, H. Burkhard, B.Bartsch-Spörl, S.Wess (eds.) Series: Lecture Notes in Computer Science 1400 Publisher: Springer-Verlag Berlin Heidelberg, Year: 1998 ISBN: 3540645721,9783540645726

2. K.Anastasi, L.M.Currie. G.H.Kim.:"Understanding diagnositic reasonging in TCM practice: tongue diagnosis" may 2009, Alternative therapies in thealth and medicine 15(3):18-28 
3. G.Wallner,F.L.Lan, M.J.Jandl.: "The way of Thinking in Chinese Medicine-Theory,methodology and Structure of Chinese Medicine" p26-27, 2010, PETER LANG Internationaler Verlag der Wissenschaften. ISBN 978-3-631-61196-8

4. Cui, H.Li, X.Hu, L.Jia,S.Yang.:" Imagery and Traditional Chinese Medicine" Chinese Journal of Library and Information Science for Traditional Chinese Medicine, Juni.2014 Vol.38 No.3.

5. Yang, et.al. "Clinical decision support system on basis of case based reasoning for Traditional Chinese Medicine" World Science and Technology/Modernization of Traditional Chinese Medicine and Materia Medica, 2014 Vol. 16,No 3.

6. De,et.al.:"Application of case-based reasoning technology in distributed system of TCM medical cases"[J]World science and Technology (modernization of traditional Chinese Medicine) 2009,11(05):698-701

7. Wang,et.al.:" research of chinese medicine expert system integrated CBR and fuzzy rule-based reasoning" journal of Yunan university.2008 Vol.30,S2:195-197,CN53-1045/ISSN 0258-7971.

8. World Health Organization.: "Global report on psoriasis" 2016 who library Cataloging in Publication Data, ISBN 9789241565189.

9. Fan, Y.Yan, Y.Zeng, Q.Tian,"Treatment according to syndrome differentiation and external use medicine in the treatment of psoriasis" Clinical observation of Shaanxi traditional Chinese medicine, 2010, Vol 31, No 8

10. Li, X.Sun, "Traditional Chinese Medicine treatment of psoriasis vulgaris" 575 cases of efficacy analysis, Jilin Chinese medicine,2010.8, Vol.30. No.8

11. Li, “Dialectic treatment of psoriasis, Chinese General Practice”, June 2005,Vol 8, No 12.

12. Zhou.:"Research and application of Chinese Medical Diagnosis Expert System" Huazhong University of Science and Technology 2005.

13. Riaño,et al. "An ontology-based personalization of health-care knowledge to support clinical decisions for chronically ill patients" Journal of Biomedical Informatics 45(2012) 429-446

14. Martinez-Romero, et al. "The iOSC3 system: using ontologies and SWRL rules for intelligent supervision and care of patients with acute cardiac disorders". Computational and mathematical methods in medicine, 2013.

15. -T.Bau, R.-C. Chen, C.-Y.Huang, "Construction of a clinical decision support system for undergoing surgery based on domain ontology and rules reasoning". TELEMEDICINE and e-HEALTH,20(5),460472. 2014

16. Alfonse, M.M.Aref, A.-B.M.Salem, "An ontology based system for cancer diseases knowledge management". International Jounal of Information Engineering and Electronic Business,6(6),55. 2014

17. Herre," General Formal Ontology (GFO): A Foundational Ontology for Conceptual Modelling. In Theory and Applications of Ontology" : Computer Applications. Edited by Poli R, Healy M, Kameas A. Netherlands: Springer; 2010:297-345. 
18. Herre, B.Heller, P.Burek, R.Hoehndorf, F.Loebe, H.Michalek, "General Formal Ontology (GFO): A Foundational Ontology Integrating Objects and Processes". Part I: Basic Principles (Version 1.0). Onto-Med Report. Research Group Ontologies in Medicine (Onto-Med), University of Leipzig; 2006.

19. Long,Y.Zhu,L.Jia,et.al.:"An ontological framework for the formalization,organization and usage of TCM-Knowledge" [J]. BMC Medical Informatics and Decision Making, 2019, 19(S2).

20. Long,Y.Zhu,et.al.:"Outline of the Construction and Application of a GFO-based TCM Diagnoses Ontology for Syndrome Differentiation of Psoriasis Vulgaris" $201719^{\text {th }}$ International Conference on E-health Networking,Application\&Services (HealthCom):The $1^{\text {st }}$ IEEE International Workshop on Artificial Intelligence in Chinese Medicine Workshop(AI\&TCM 2017) ISBN

21. China Association of Traditional Chinese Medicine Department of Dermatology, "psoriasis vulgaris (white crust) TCM evidence-based clinical practice guidelines", Journal of traditional Chinese medicine, January 2014, Vol 55, No 1.

22. Psoriasis Committee of Dermatology and Venereology branch of Chinese Medical Association.:"China Psoriasis Diagnosis and Treatment Guide” [J] Chinese Journal of Dermatology,April 2019.Vol.52,No.4

23. Friedman,A.Kandel.:"Introduction to pattern recognition statistical,structural,neural and fuzzy logic approaches" series in Machine perception artifical intelligence Vol.32, 1999. published by Imperial college press 57 shelton street, covent garden,London.ISBN 9810233124

24. -J.Zimmermann.:"Fuzzy Set Theory-and Its Applications"- Fourth Edition,2011 Kluwer Academic Publishers.

25. A.Zadeh.:"Fuzzy Sets" Information and Control,8, 338-353 (1965)

26. A.Zadeh.:"outline of a new approach to the analysis of complex systems and decision processes" IEEE transaction on system,man,and cybernetics,Vol.SMC-3,No.1 Jan 1973

27. A.Zadeh.:"Fuzzy logic and approximate reasoning" Synthese,30,407

28. Z.Wang,L.Y.Han.:"Applied fuzzy mathematics" 1989 Press of Capital University of Economics and Trade ISBN 7-5638-0129-4/0.3

29. Uciteli, C. Beger, T. Kirsten, F.A. Meineke,and H. Herre 2019. Ontological Modelling and Reasoning of Phenotypes Proceedings of the Joint Ontology Workshops (JOWO) 2019, Episode V: The Styrian Autumn of Ontology, Graz, Austria, 2019, CEUR Workshop Proceedings, Vol. 2518, 2019 CEUR Workshop Proceedings. Vol. 2518, [BibTeX] [http://ceur-ws.org/Vol-2518/paper-ODLS11.pdf]

\section{Figures}




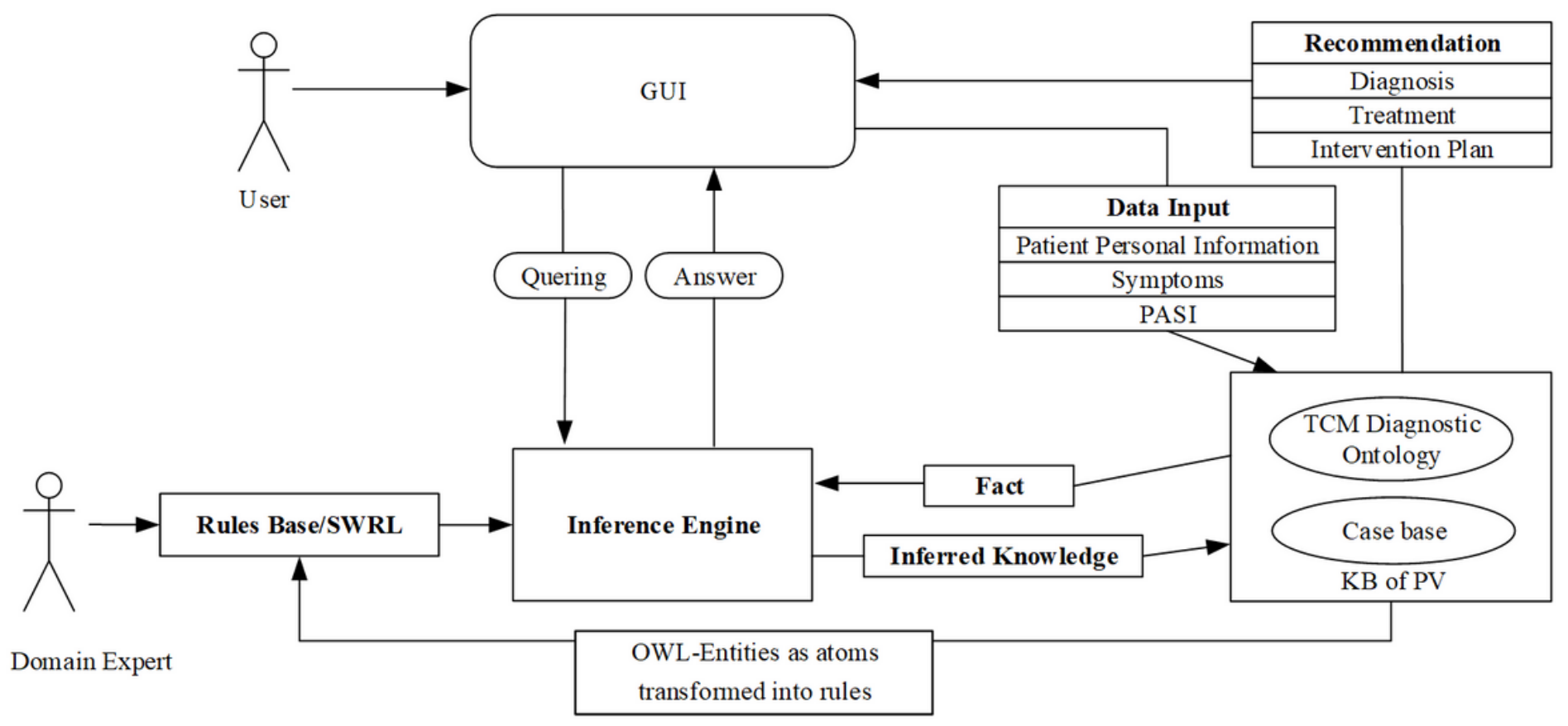

Figure 1

Architecture of ONTOPV system 


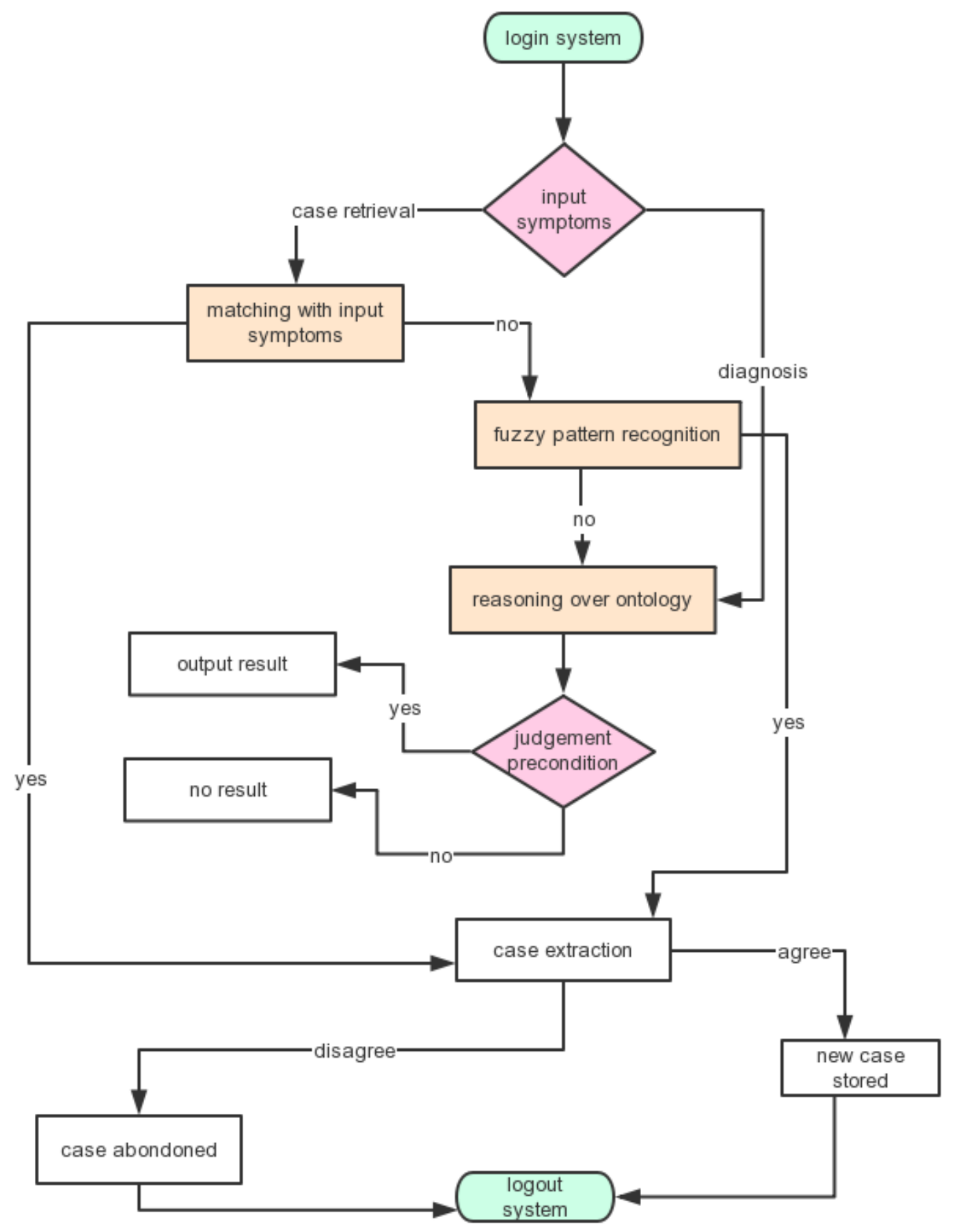

Figure 2

Workflow of case retrieval in ONTOPV prototype 


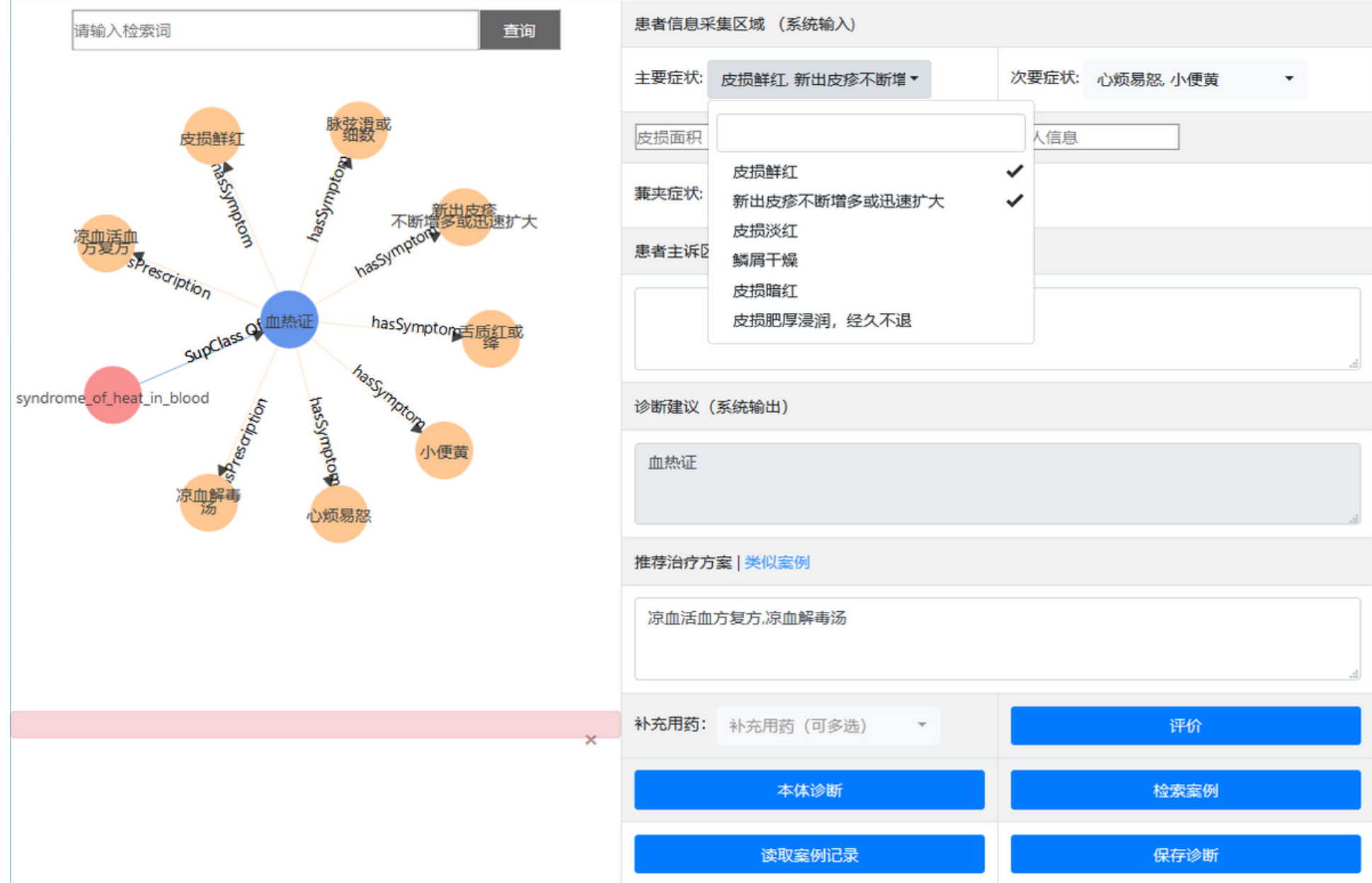

\section{Figure 3}

An example of ontology reasoning 


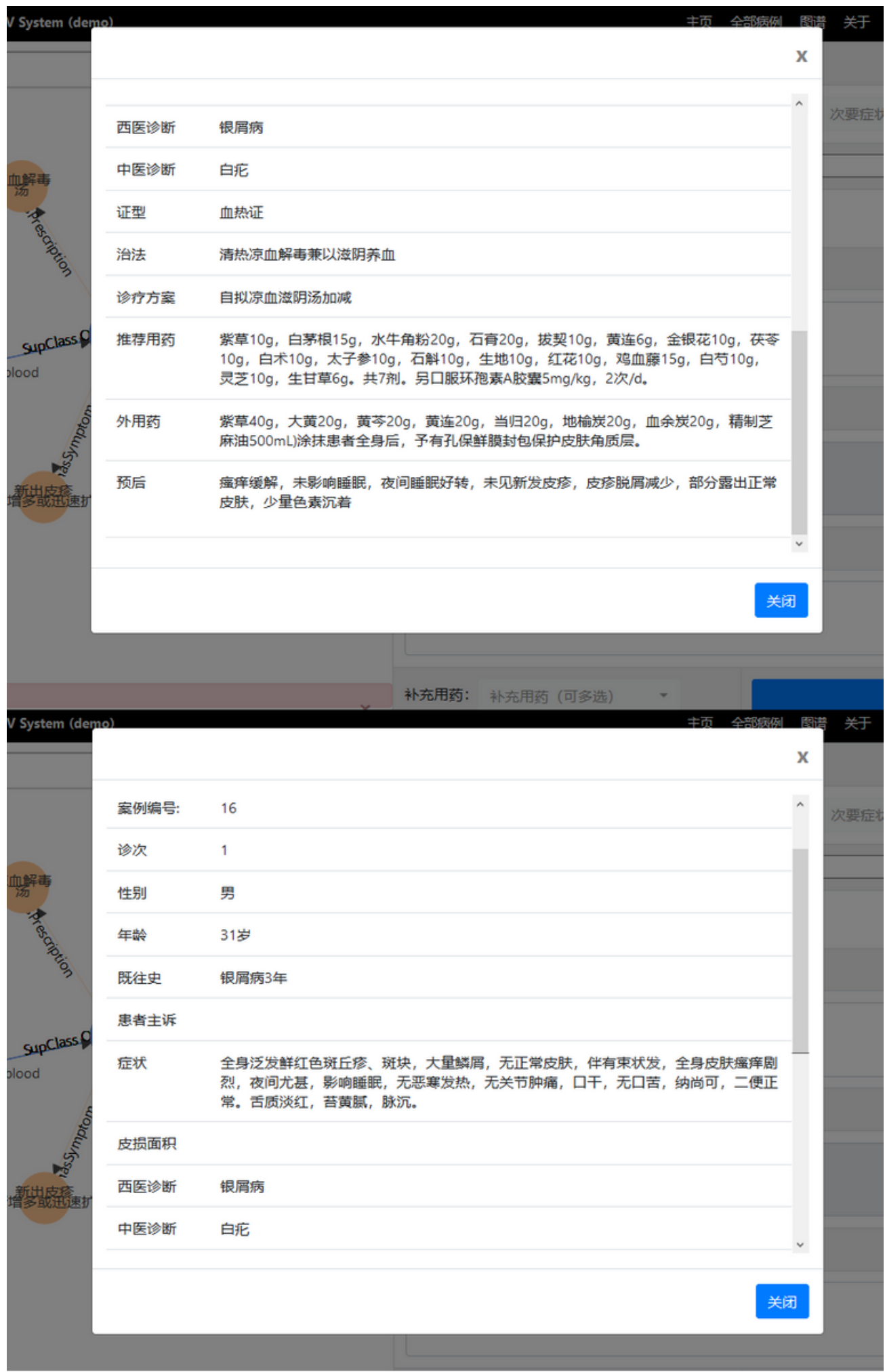

\section{Figure 4}

An example of case retrieval using fuzzy pattern recognition 
请输入检索词

查询

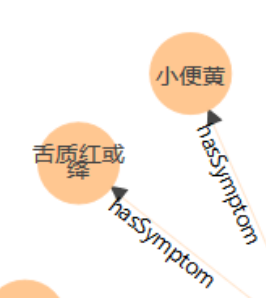

\section{凉血解毒}

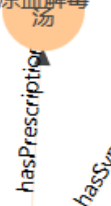

心烦易怒

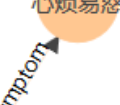
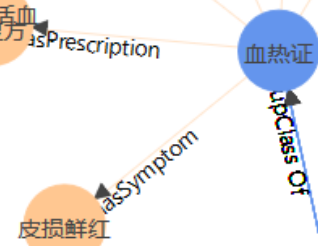<smiles></smiles>
nas 5

新出皮凃

不断䏆岁或逊速扩大

年的红

$$
\text { syndrome_of_heat_in_blood }
$$

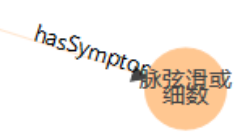

胃舒平和紫丹银首胶囊有不良反应！

$\times$

血热证皮损鲜红、新出皮疹增多时不宜药浴

$\times$
患者信息采集区域（系统输入）

主要症状: 主要症状 (可名选)

皮损面积

次要症状: 次要症状 (可多选)

个人信息

萐夹症状: 䓦夹症状 (可多选)

患者主诉区

诊断建议 (系统输出)

血热证

推荐治疗方案 | 类似案例

凉血活血方复方, 凉血解毒汤, 紫丹银屏胶喪, 胃舒平

补充用药：胃舒平

评价

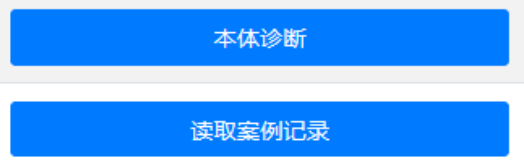

检索室例

保存沴断

\section{Figure 5}

An example of semantic query 


\section{OntoPV System (demo)}

凉血解专汤

查询

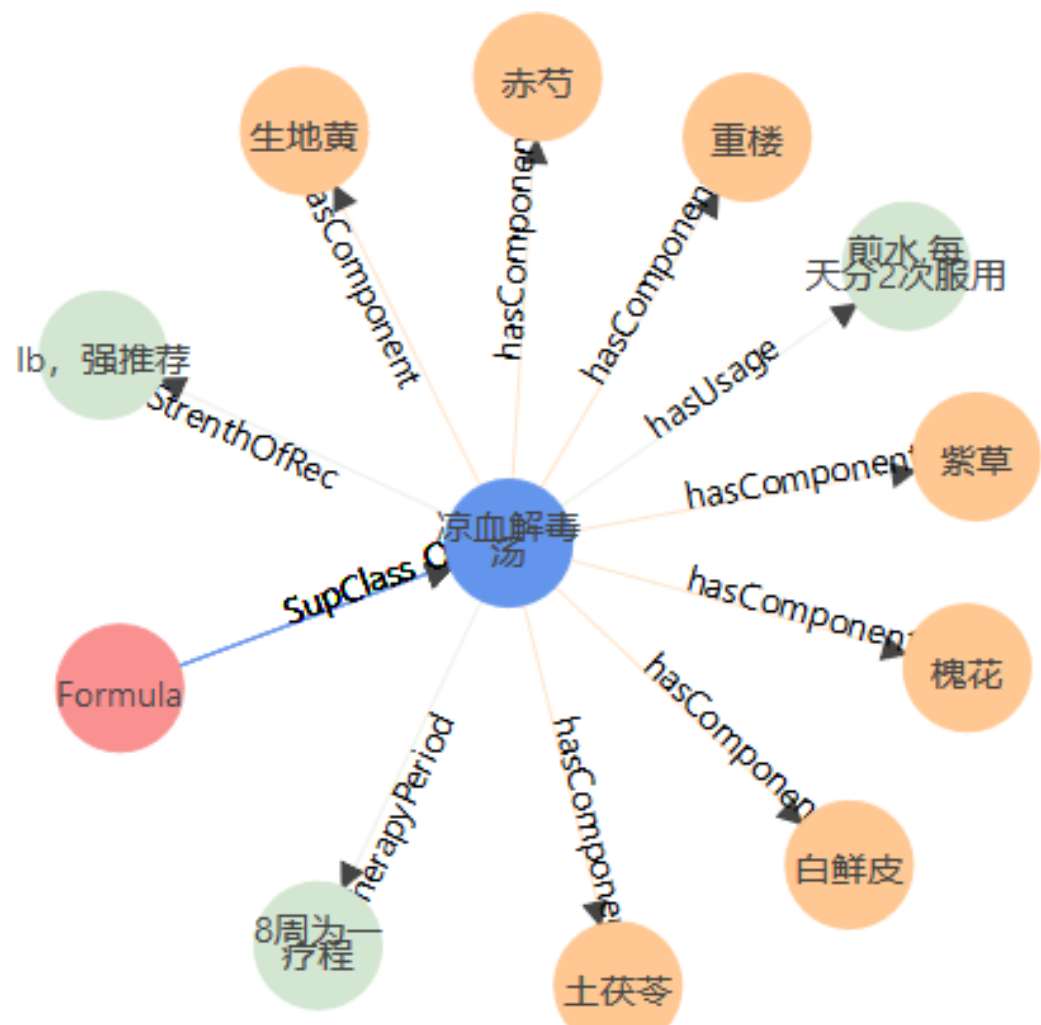

凉血解毒汤: 土获苓 $30 \mathrm{~g}$, 槐花 $15 \mathrm{~g}$, 紫草 $10 \mathrm{~g}$, 重楼 $9 \mathrm{~g}$, 生地黄 $15 \mathrm{~g}$, 白鲜皮 $\times$ $10 \mathrm{~g}$ ，赤苛 $10 \mathrm{~g}$. 每天一剂，浓煎后分 2 次服用，共7剂。

\section{Figure 6}

An example of medication alert 